Christopher Pollmann

\title{
Der Vertreter der Länder in Angelegenheiten der Europäischen Union - Schutz des Föderalismus oder Beruhigungspille?
}

Bekanntlich wurde zur Ratifizierung des Vertrags von Maastricht auch ein EuropaArtikel in das Grundgesetz eingefügt, Art. 23. Die sieben Absätze dieses Textes zielen wesentlich darauf ab, die Mitwirkung der Länder in Angelegenheiten der Europäischen Union zu stärken. Zu diesem Zweck gewährt Art. 23 GG dem Bundesrat Informations- und Beteiligungsrechte gegenüber der Bundesregierung; auf der europäischen Ebene soll außerdem - und das ist besonders neuartig - in Bereichen ausschließlicher Landesgesetzgebung ein Vertreter der Länder für Deutschland sprechen. Es handelt sich dabei in der Praxis um einen Ländervertreter je betroffenem Sachgebiet, wobei die Bundesländer sich bei der Wahrnehmung dieser Aufgabe abwechseln.

Die neuen Regelungen haben recht unterschiedliche Reaktionen erfahren. Die Länder sehen in ihnen einen großen Fortschritt zum Schutz des deutschen Föderalismus und bei der Vertretung ihrer Interessen. Juristen und Politologen äußern hingegen erhebliche Zweifel an der Rechtmäßigkeit, der Wünschbarkeit und der Praktikabilität der neuen Bestimmungen. Vor diesem Hintergrund möchte ich die Frage aufwerfen, inwieweit der Ländervertreter tatsächlich zur Erhaltung der Vielfalt und der Eigenständigkeit der Bundesländer beiträgt. Oder wird den Ländern hier eine Beruhigungspille verabreicht, damit die weitere Aushöhlung des Föderalismus durch die europäische Integration bekömmlich bleibe?

Aus der Literatur sind kaum praktische Erfahrungen mit den neuen Regelungen bekannt. ${ }^{I}$ Um die eben gestellte Frage - vorläufig - zu beantworten, sind deshalb einige theoretische Überlegungen erforderlich. So läßt sich in einem ersten Teil zeigen, daß der Ländervertreter in Wahrheit als Vertreter des Bundes handelt. Im zweiten Teil wird sich dann herausstellen, daß es auch höchst schwierig ist, die Interessen aller Länder zur Geltung zu bringen. Der dritte Teil wird schließlich deutlich machen, daß Ländervertreter und Bundesrat letztlich dazu beitragen, die Zentralisierung von Länderkompetenzen zu legitimieren.

\section{Der Ländervertreter als Vertreter des Bundes}

Art. 23 GG spricht vom >Vertreter der Länder . Aber nirgends wird deutlich, wie sichergestellt werden kann, daß diese Person auch tatsächlich die Interessen der Länder zum Ausdruck bringt. Im Gegenteil, sowohl die offiziell normierte Rolle des Ländervertreters (1) wie auch seine institutionelle Anbindung an den Bund (2) begründen den Verdacht, daß der Ländervertreter paradoxerweise die Interessen des Bundes vertreten soll.

\footnotetext{
I Die einzigen, recht allgemeinen Hinweise in diese Richtung habe ich angetroffen im außerst knappen Bericht von Hans Hoffmann u. Klaus G. Meyer-Teschendorf, Der "Europaartikel" $23 \mathrm{GG}$ in der staatlichen Praxıs, ZG 1997, S. $86 \mathrm{ff}$.
} 


\section{Die Rolle des Ländervertreters}

Art. 23 VI I GG bestimmt: , Wenn im Schwerpunkt ausschließliche Gesetzgebungsbefugnisse der Länder betroffen sind, soll die Wahrnehmung der Rechte, die der Bundesrepublik Deutschland als Mitgliedstaat der Europäischen Union zustehen, vom Bund auf einen vom Bundesrat benannten Vertreter der Länder übertragen werden.< Der Ländervertreter hat also in den Unionsgremien die Rechte von ganz Deutschland und nicht etwa die Rechte der einzelnen Länder wahrzunehmen. Art. 23 VI 2 (2. Hs.) GG spricht das auch offen aus, indem er den Ländervertreter verpflichtet, >die gesamtstaatliche Verantwortung des Bundes zu wahren . Diese schwere Aufgabe wird ihm dann auch nicht von den Ländern, sondern "vom Bund « übertragen.

Art. 23 VI GG konkretisiert Art. I46 I des EWG-Vertrages, der durch den Maastrichter Vertrag erweitert wurde. Früher hieß es dort im Hinblick auf den Rat der Europäischen Gemeinschaften: ,Der Rat besteht aus Vertretern der Mitgliedstaaten. Jede Regierung entsendet eines ihrer Mitglieder. $`$ In seiner neuen Fassung lautet Art. I 46 I: ,Der Rat setzt sich je Mitgliedstaat aus einem Vertreter auf Ministerebene zusammen, der befugt ist, für die Regierung dieses Mitgliedstaates verbindlich zu handeln. Es muß sich zwar nach wie vor um eine Person mit Ministerstatus handeln. Aber sie muß nicht mehr notwendig der Zentralregierung angehören. Der Zweck dieser Änderung liegt darin, den Ministerrat für die Vertreter von Regionen, Bundesländern, Autonomen Gemeinschaften u. ä. zu öfnen. Art. 23 VI GG hat diese Mög]ichkeit verwirklicht. Jedoch soll sich nichts daran ändern, daß die entsprechenden Personen den jeweiligen Mitgliedstaat in seiner Gesamtheit zu vertreten haben. Das klingt ja auch selbstverständlich, berechtigt uns aber zu der Frage, ob sie wirklich befugt und in der Lage sind, die Interessen von Regionen, Bundesländern o. ä. zum Ausdruck zu bringen.

Diese Frage stellt sich noch verstärkt angesichts der verfahrensmäßigen Ausgestaltung des Ländervertreters.

\section{Die institutionelle Anbindung des Ländervertreters an den Bund}

Wie bereits festgestellt, wird der Ländervertreter vom Bundesrat benannt und nicht etwa durch eine Versammlung von Landesparlamentariern oder Landesregierungen. Der Bundesrat aber ist angelegt als ein Organ des Bundes und nicht als bloße Interessenvertretung der Länder. Bei Ausarbeitung des Grundgesetzes wurde nämlich mit Bedacht das amerikanische Senatsmodell verworfen, wonach jeder Gliedstaat mit einer Stimme ausgestattet ist, um seine spezifischen Interessen auf Bundesebene geltend zu machen. ${ }^{2}$

Die Anbindung an den Bund ergibt sich noch deutlicher aus Art. ${ }_{3}$ VI 2 (I. Hs.) GG: ,Die Wahrnehmung der Rechte (nämlich Deutschlands als Mitgliedstaat der Europäischen Union) erfolgt unter Beteiligung und in Abstimmung mit der Bundesregierung $(. .)<$., wobei »Abstimmung « mehr als "Benehmen « und weniger als »Einvernehmen « heißen soll. ${ }^{3}$ Diese subtile Vorschrift ist im Zusammenhang mit Art. $23 \mathrm{~V}$ 2 GG und dem zu Art. 23 V und VI GG ergangenen Ausführungsgesetz ${ }^{4}$ zu lesen. Stimmen die Auffassungen von Bundesrat und Bundesregierung nicht überein und

2 Vgl. BVerfGE 8, xO4 ff. (I 20f.).

3 Vgl. die Gesetzesbegrundung der Bundesregierung und die Stellungnahme des Bundestagsausschusses fur Forschung, Techrologie und Techmikfolgenabschatzung zur Einfugung des Art. 23 , Drs. I2/3338, S. 10 , sowie $12 / 3896$, S. 20.

4 V. I2.3. 1993, BGBl. r993 I, S. 3 I 3 ff. 
kommt keine Einigung zustande, so ist die Auffassung des Bundesrates maßgebend, wenn er sie mit $^{2 / 3}$-Mehrheit bestätigt ( $\$ S$ II). Zwar kommt dem Ländervertreter also womöglich das letzte Wort zu. Doch die ihm auferlegte Abstimmung mit der Bundesregierung ermöglicht dieser, kraft ihrer Erfahrung und ihres Überblicks in Europaangelegenheiten Druck auf den Ländervertreter auszuüben. Sie wird ihn auch aus Statusgründen beeinflussen können, wie wir gleich sehen werden.

Der bereits zitierte Art. 146 I EG-Vertrag verlangt, daß Ratsmitglieder befugt sein müssen, für die Regierung ihres Staates verbindlich zu handeln. Das gilt sowohl für die Ministerräte als auch für die vorbereitenden Sitzungen auf Beamtenebene. Die Minister oder Beamten einer Landesregierung mögen zur Vertretung von Gesamtdeutschland befugt sein, dennoch haben sie meistens wohl nicht die gleiche Autorität wie die Mitglieder oder Mitarbeiter einer Zentralregierung. Die parlamentarische Legitimation des Ländervertreters über den Bundesrat ist im übrigen höchst indirekt.

Die französischen oder irischen Ratsmitglieder etwa werden sich also unweigerlich fragen, ob ein deutscher Landesminister nicht nur das Recht, sondern auch das Gewicht hat, die Bundesrepublik Deutschland zuverlässig zu binden. Um solchen Bedenken zuvorzukommen, wird der Ländervertreter den Konflikt mit der Bundesregierung meiden, zumindest alles dafür tun, damit eine Meinungsverschiedenheit nicht den Vertretern der anderen Mitgliedstaaten zu Ohren kommt. Das dürfte nicht immer leicht sein, zumal die Bundesregierung den Ländervertreter durch Offenlegung von Differenzen gezielt unter Druck setzen könnte. Selbst im Falle seines Letztentscheidungsrechts befindet sich der Ländervertreter gegenüber der Bundesregierung mithin in einer tendenziell schwachen Position. Soweit die Bundesregierung sich sperrt, ist der Schutz der Länderinteressen in der Europäischen Union daher schwierig.

Das Letztentscheidungsrecht des Ländervertreters erfährt aber auch eine textsystematische Einschränkung. Art. $23 \mathrm{~V}_{3}$ GG bestimmt, daß Entscheidungen, die Ausgabenerhöhungen oder Einnahmeminderungen für den Bund bewirken können, der Zustimmung der Bundesregierung bedürfen. Auch das Handeln der Europäischen Union kostet regelmäßig Geld, und es fragt sich daher, was vom Letztentscheidungsrecht des Ländervertreters unter diesen Umständen noch übrig bleiben wird.

Der Ländervertreter ist nicht nur in seiner juristischen Ausgestaltung auf die Vertretung des Bundes verpflichtet. Er dürfte darüberhinaus kaum in der Lage sein, die Interessen aller Länder zu vertreten.

\section{Die schwierige Interessenvertretung aller Länder}

Der Ländervertreter steht zunächst einmal vor dem Problem, daß die allen Bundesländern gemeinsamen Interessen in der europapolitischen Auseinandersetzung wahrscheinlich von geringem Gewicht sind, zumal Art. 23 VI GG nur hinsichtlich der stark begrenzten »ausschließlichen Gesetzgebungsbefugnisse « der Länder ${ }^{5}$ gilt ( 1 ). Auch aus diesem Grunde entscheidet der Bundesrat mit Mehrheit, wenn es um die Benennung und Instruktion des Ländervertreters geht. Das ist verfassungsrechtlich problematisch, aber praktisch unumgänglich (2). Der Ländervertreter erhält also ein imperatives Mandat, ebenso wie die Mitglieder des Bundesrates an die Weisungen ihrer jeweiligen Landesregierungen gebunden sind. Diese imperativen Mandate gel-

\footnotetext{
$5 \mathrm{Zu}$ dieser, ansonsten nicht im Grundgesetz vorgesehenen Kategorie, vgl. die Gesetzesbegründung (Fn. 3),
} S. 9 


\section{Die geringe Bedeutung der allen Ländern gemeinsamen Interessen}

Die den Bundesländern gemeinsamen europapolitischen Interessen lassen sich in drei Kategorien einteilen. Am häufigsten ist wohl das Anliegen, die Regelung eines bestimmten Sachverhalts durch die Europäische Union schlicht zu verhindern, sei es, um die eigenen, also Landeskompetenzen in dem betroffenen Bereich zu erhalten, sei es, um zusätzlichen finanziellen Belastungen aus dem Weg zu gehen. Diese bloßen $\Lambda$ bwehrinteressen dürften meist nicht durchsetzbar sein. Zum einen kann die Bundesrepublik im Rat überstimmt werden. Zum anderen mag auch der Bundesregierung an der fraglichen Regelung gelegen sein. Denn das Tätigwerden der Europäischen Union gibt ihr die Gelegenheit, indirekt auch auf die Verhältnisse in den anderen Mitgliedstaaten einzuwirken. Sobald die Bundesländer nun statt einer unrealistischen Abwehrhaltung den Versuch unternehmen, die Ausformulierung der vorgeschlagenen Regelung zu beeinflussen, z. B. um ihren Anwendungsbereich möglichst klein zu halten, dürften ihre Interessen aber auseinandergehen, je nach Zusammensetzung der Bevölkerung, geographischer und wirtschaftlicher Lage sowie politischer Orientierung der einze]nen Länder.

Die zweite Kategorie der allen Ländern gemeinsamen Interessen beruht auf der Annahme, daß die Europäische Union den Regionen und in Deutschland den Bundesländern finanzielle oder andere Leistungen gewähren will, z. B. Geld für Aufsatzwettbewerbe an Schulen. Die Länder würden dem Vorhaben wahrscheinlich einhellig zustimmen, während die Bundesregierung es vielleicht aus übergreifenden Erwägungen ablehnt, etwa weil sie längerfristig eine gewisse Abhängigkeit der Länder von der Union befürchtet. Diese Interessenkonstellation dürfte nur selten auftreten.

Die dritte Fallgruppe umfaßt die restlichen von allen Ländern geteilten Interessen. Es ist davon auszugehen, daß es sich dabei um Interessen handelt, die der Position des Bundes und der Bundesregierung nahe kommen oder gar mit ihr übereinstimmen. Daraus folgt, daß der Ländervertreter insoweit nicht besonders gefordert ist.

Aus dieser Interessenanalyse ergibt sich folgendes: Die allen Ländern gemeinsamen Interessen sind kaum durchsetzbar oder bedürfen keiner speziellen Vertretung gegenüber der Bundesregierung. Die realisierbaren Anliegen der Länder sind also meist verschiedenartig, ja widersprüchlich. Soweit der Ländervertreter überhaupt in der Lage ist, seiner Verpflichtung auf das Bundesinteresse zu entgehen, wird er daher kaum die Interessen aller Länder in ihrer Vielfalt und Unterschiedlichkeit zum Ausdruck bringen können. Er wird in der Regel allenfalls die Position einer Ländermehrheit vertreten können.

\section{Die verfassungsrechtlich problematische, aber praktisch unumgängliche Anwendung des Mebrheitsprinzips}

Der Ländervertreter wird vom Bundesrat benannt und instruiert. Der Bundesrat beschließt dabei mit Stimmenmehrheit. Das ergibt sich nicht direkt aus dem neuen Art. $23 \mathrm{GG}$, sondern aus Art. 52 III I GG, der hier mangels anderweitiger Regelung anzuwenden ist. Was sich so selbstverständlich anhört, ist im Hinblick auf die Autonomie der Länder recht bedenklich. Das Problem besteht darin, daß ein Ländervertreter nur dann benannt wird, wenn ausschließliche Gesetzgebungsbefugnisse der Länder betroffen sind. In diesen Bereichen sind weder der Bund noch eine Mehrheit 
von Ländern befugt, einem einzelnen Land Vorschriften zu machen. Genau das

könnte die Anwendung der Mehrheitsregel im Bundesrat aber mit sich bringen, wenn ein Land überstimmt wird. Ist es daher verfassungsrechtlich geboten, Entscheidungen einstimmig zu treffen?

Das Erfordernis der Einstimmigkeit im Bundesstaat soll insbesondere die Freiheit eines jeden Gliedstaates und seiner Bürger sichern. ${ }^{6}$ Diese Funktion geht jedoch verloren, sobald die Bundesländer aufgefordert sind, eine einheitliche Stellungnahme zu einem Vorhaben der Europäischen Union abzugeben. Das liegt daran, daß das Handeln der Europäischen Union - im Gegensatz zu einem Tätigwerden der Ländergemeinschaft - nicht yon einem positiven Votum der Länder abhängt. Auch wenn die Länder sich also mangels Einstimmigkeit nicht auf eine Position verständigen, kann die Union trotzdem handeln. Die Freiheit der einzelnen Länder zur Wahrnehmung ihrer Belange wird insoweit eingeschränkt, und zwar auf Dauer. Eine spätere Einigung vermag diesen Eingriff nicht wieder rückgängig zu machen. Darüberhinaus versagen sich die Länder so jegliche Einflußnahme auf das Handeln der Union. Gegenüber den Vorhaben der Europäischen Union hat das Einstimmigkeitserfordernis somit keine freiheitsschützende, sondern letztlich eine freiheitsbeeinträchtigende Wirkung.

Diese Überlegungen sind nicht etwa hypothetischer Art. Von 1979 bis zur Einheitlichen Europäischen Akte von 1986 konnten die Länder sich nämlich auf Basis der Einstimmigkeit zu EG-Vorhaben äußern. In diesem Zeitraum haben sie nur 37 Stellungnahmen erarbeitet, von denen nur eine einzige an die Bundesregierung weitergeleitet wurde, und dies auch noch auf deren eigene Veranlassung.? Das Gesetz zur Einheitlichen Europäischen Akte (EEAG) ${ }^{8}$ regelte die Mitwirkung der Länder hingegen über den - mit Mehrheit entscheidenden - Bundesrat. Das führte zu einem grundlegenden Wandel: So hat der Bundesrat von 1987 bis 1990769 Stellungnahmen zu EG-Angelegenheiten abgegeben.?

So erscheint es denn vertretbar, daß die Länder ihre Haltung in Fragen der Europäischen Union auch dann mit Mehrheit festlegen, wenn ihre ausschließlichen Gesetzgebungsbefugnisse betroffen sind. ${ }^{\circ}$ Das bedeutet aber zugleich, daß nicht die Anliegen aller Länder zum Ausdruck gebracht werden können, sondern gewissermaßen nur der gemeinsame Nenner bzw. die Mehrheitsinteressen. Die föderale Vielfalt bleibt dabei auf der Strecke.

\section{Probleme mit dem imperativen Mandat}

Die Mitglieder des Bundesrats unterliegen gemeinhin einem imperativen Mandat ihrer jeweiligen Landesregierung, aber nicht des Landesparlaments (a). Darüberhinaus stellen sich Fragen nach der Zulässigkeit und Praktikabilität der Weisungsgebundenheit des Ländervertreters an den Bundesrat (b).

a) Wenn Vertreter der I6 Landesregierungen zusammentreffen, um eine Stellungnahme zu einem Vorhaben der Europäischen Union zu erarbeiten und einen Länder-

\footnotetext{
6 Vgl. BVerfGE I, 299 ff. (31 2-315).

7 Vgl. Rudolf Morawitz, Die Zusammenarbett von Bund und Landern bet der Wabrnebmung von EGAufgaben. Erfabrungen und Reformbestrebungen, in: S. Magiera / D. Merten (Hg.), Bundeslander und Europäische Gemeinschaft, 1988, S. 45 ff. (46-48); Hermann-Josef Blanke, Foderalismus und Integratwonsgewalt. Die Bundesrepublik Deutscbland, Spanten, Italien und Belgien als dezentralisterte Staaten in $\operatorname{der} E G$, 1991, S. 270 f., Fn. 49 u. 54. u. a. (Hg.), Die deutschen Lander in Europa. Politische Union und Wirtschafts- und Währungsunion, 1992, S. 171 ff. (175).

io So auch uberzeugend Blanke (Fn. 7), S. $406 \mathrm{f}$.
} 
vertreter zu benennen, so werden bei fehlender Einstimmigkeit häufig nicht nur eine oder mehrere Delegationen überstimmt. Darüberhinaus müssen in der Regel auch andere Delegationen Abstriche von ihren Positionen machen, um Kompromisse und die Bildung einer Mehrheit zu ermöglichen. Das dürfte nicht immer einfach sein, verbleibt aber meist im Dunkel der Regierungsinterna.

Es läßt sich leicht denken, daß die Probleme erheblich zunehmen würden, wenn die Mitglieder des Bundesrates auch oder gar in erster Linie an Weisungen der Landesparlamente gebunden wären. Das Bundesverfassungsgericht hatte 1958 die lediglich konsultativen Volksbefragungen zur Atombewaffnung für verfassungswidrig erklärt, weil sie einen unzulässigen Druck auf die Vertreter der beiden Landesregierungen im Bundesrat ausübten. ${ }^{I I}$ Weisungen stünden demnach mit dem Grundgesetz erst recht in Widerspruch. Jedoch ist fraglich, ob diese Prinzipien der vertikalen Gewaltenteilung auch heute noch in der damaligen Strenge gelten, insbesondere im Verhältnis zur europäischen Ebene. Seit 1958 haben die Landesparlamente zahlreiche Kompetenzen an den Bund und an die Europäischen Gemeinschaften verloren. Die Substanz des Föderalismus erscheint in Gefahr. Deshalb wird heute zunehmend erwogen, den Landesparlamenten ein Weisungsrecht gegenüber den Mitgliedern des Bundesrats einzuräumen, zumindest in Angelegenheiten der Europäischen Union. ${ }^{12}$ Ich möchte den Vorschlag hier nicht vertiefend behandeln, sondern nur daraufhinweisen, daß die Abstimmung zwischen den Bundesratsmitgliedern und den Ländern schwieriger würde.

b) Das imperative Mandat des Ländervertreters wirft schon heute gewichtige Fragen auf. Vor allem wird die Besorgnis geäußert, die Verhandlungs- und Kompromißfähigkeit Deutschlands in der Europäischen Union sei beeinträchtigt, wenn die deutsche Delegation im Rat und in anderen Gremien einem imperativen Mandat unterliege. Es könne dann auch leicht passieren, daß sie einfach überstimmt werde. ${ }^{13}$ Schwierigkeiten gebe es insbesondere, wenn Paketlösungen zur Verhandlung anstünden. ${ }^{14}$

Vor diesem Hintergrund wird verschiedentlich behauptet, die Weisungsgebundenheit des Ländervertreters mindere die Entscheidungsfähigkeit der Unionsorgane und sei deshalb europarechtswidrig. Sie verstoße namentlich gegen das Gebot des gemeinschaftsfreundlichen Verhaltens in Art. 5 des EG-Vertrags. ${ }^{15}$

Auch innerstaatlich wird an der Rechtmäßigkeit dieses imperativen Mandats gezweifelt. Das Bundesverfassungsgericht hat nämlich in anderen Zusammenhängen der Flexibilität der Bundesregierung in der Außenpolitik Verfassungsrang zugesprochen. ${ }^{16}$ Abgesehen davon, daß die Angelegenheiten der europäischen Integration häufig bereits einen quasi-innenpolitischen Charakter aufweisen, ließen sich alldem die Prinzipien des Föderalismus und der demokratischen Kontrolle entgegenhalten. Das Problem soll hier nicht vertieft, geschweige denn gelöst werden, weil das Mandat des Ländervertreters sowieso nicht sonderlich imperativ sein wird.

Zunächst einmal, wie bereits festgestellt, besitzt der Ländervertreter gegenüber der

II BVerfGE 8, S. I04 ff. (I 20 f.).

12 Vgl. Michael Schweitzer, Betelligung der Bundeslander an der europasschen Gesetzgebung, ZG 1992, S. I 28 ff. (1 47 f. m. w. N.).

13 Vgl. zu beidem Ulrich Everling, Uberlegungen zur Struktur der Europalschen Unıon und zum neuen Europa-Artzkel des Grundgesetzes, DVBI. 1993, S. $936 \mathrm{ff}$. (947); Werner von Simson / Jürgen Schwarze, Europasche Integration und Grundgesetz. Maastricht und die Folgen fur das deutschen Verfassungsrecht, 1992, S. $38 \mathrm{f}$.

14 Vgl. Albrecht Randelzhofer, Fragenkatalog zu der Anhörung der Gemeinsamen Verfassungskommission von Bundestag und Bundesrat am 22. 5. 1992, „Grundgesetz und Europa" (Arbeitsunterlage Nr. 34), S. 6.

Is So Everling (Fn. 13), S. 946; Rùdiger Breuer, Die Sackgasse des neuen Europaartikels (Art. ${ }_{23}$ GG), NVwZ I 994, S. 417 ff. (427).

16 Vgl. BVerfGE 80, S. 74 ff. (8of.); 68, S. x ff. (83 ff./87). 
Bundesregierung nur bei einer 2/3-Mehrheit im Bundesrat das letzte Wort. Selbst wenn dem Ländervertreter danach das Letztentscheidungsrecht zustünde, dürfte er große Schwierigkeiten haben, seine Position gegen die Bedenken der Bundesregierung auch auf europäischer Ebene durchzusetzen. In den Verhandlungen mit is anderen Mitgliedstaaten wird er auf zahlreiche weitere Forderungen und Anliegen sowie äußerst komplexe Zwänge stoßen. In einem solchen Kontext könnte seine Weisungsgebundenheit kontraproduktiv wirken. Empirische und experimentelle Untersuchungen haben jedenfalls gezeigt, daß die Chancen erfolgreicher Verhandlungen in dem Maße sinken, wie die Bindung der Verhandlungsführer an die Weisungen ihrer jeweiligen Auftraggeber zunimmt. ${ }^{17}$ Angesichts dieser Probleme ist zu vermuten, daß die dem Ländervertreter erteilten Instruktionen eher als Wünsche denn als verbindliche Weisungen verstanden werden, zumal die Länder selbst davon ausgehen, daß dem Ländervertreter Verhandlungsspielraum gelassen werden müsse. $^{8}$

Ein imperatives Mandat ließe sich auch kaum parlamentarisch oder gerichtlich durchsetzen bzw. kontrollieren. Wie sollen Parlamentarier des Bundes und der Länder ihren Kontrollaufgaben nachkommen, wenn sich zunächst die Landesregierungen im Bundesrat auf eine in Brüssel zu verfolgende Verhandlungslinie einigen, diese dann mit der Bundesregierung abstimmen und schließlich in den europäischen Gremien weitere Kompromisse geschlossen werden müssen ? $^{19}$ Obwohl der Ländervertreter befugt ist, die Bundesrepublik Deutschland zu verpflichten, ist er weder dem Bundestag noch den einzelnen Landesparlamenten verantwortlich, allenfalls dem Parlament seines eigenen Bundeslandes. Umfassende Kontrollmöglichkeiten besitzt nur der Bundesrat, da dieser ja den Ländervertreter benennt. Seine demokratische Legitimation ist aber bloß indirekt. Das alles ist schon für sich genommen höchst bedenklich im Hinblick auf das Demokratieprinzip. In unserem Zusammenhang belegt es die Schwierigkeit, ein imperatives Mandat des Ländervertreters zu praktizieren.

Selbst seine verfassungsgerichtliche Durchsetzung könnte bereits an der Zwitterstellung des Ländervertreters scheitern. Im Konfliktfall dürfte es sich hier der Sache nach meist um einen Bund-Länder-Streit (Art. 93 I Nr. 3 GG) handeln. Gemäß $\$ 68$ BVerfGG können im Bund-Länder-Streit nur die Bundesregierung und eine Landesregierung beteiligt sein. Der Bundesregierung ist das Verhalten des Ländervertreters aber wohl nicht zuzurechnen. Ein Bund-Länder-Streit scheidet daher aus.

Dann kommt nur noch der Organstreit laut Art. 93 I Nr. I GG in Betracht. Beteiligtenfähig sind u. a. der Bundesrat und seine im Grundgesetz mit eigenen Rechten ausgestatteten Teile, $\$ 6_{3}$ BVerfGG. Der Ländervertreter wird zwar in Art. 23 VI GG mit eigenen Rechten bedacht. Aber da er nicht notwendig dem Bundesrat angehören muß, stellt sich die Frage, ob er als Teil des Bundesrats zu gelten hat. Die >mit eigenen Rechten ausgestatteten Teile< eines Organs wie Bundestag oder Bundesrat meint wohl die für diese Organe konstitutiven Personen wie Präsidenten und Fraktionen, aber womöglich nicht die von diesen Organen - und sei es in ihren eigenen Reihen - ernannten selbständig handelnden Funktionsträger wie Datenschutz- und Ausländerbeauftragte und eben der Ländervertreter. Selbst wenn dem Ländervertreter die Beteiligtenfähigkeit im Organstreit zugesprochen würde, käme als Antragsteller wohl der Bundesrat, aber nicht ein einzelnes Bundesland und seine

I7 Vgl. Fritz W. Scharpf, Stellungnahme fur die Gemeinsame Verfassungskommission von Bundestag und Bundesrat zu der Anhorung am 22, 5. 1992, "Grundgesetz und Europa " (Arbeitsunterlage Nr. 3I), S. 8 f. 18 Vgl. Paul Wilhelm, Europa im Grundgesetz: Der neue Artıkel 23, BayVBI. 1992, S. 70 ff. (710).

19 Thomas Oppermann / Claus Dieter Classen, Die EG vor der Europatschen Union, NJW I993, S. 5 ff. (12); Breuer (Fn. 15), S. 428 
Regierung in Betracht. ${ }^{20}$ Ein Organstreit des Bundesrats gegen den Ländervertreter aber wäre ziemlich skurril und deshalb auch unwahrscheinlich, denn der zumeist eigentliche Gegner des Bundesrats, nämlich die Bundesregierung, käme auf diese Weise aus dem Blickfeld. Das soll keineswegs heißen, daß ein Organstreit über die Auslegung des Art. 23 VI GG nicht in Betracht kommt. Namentlich die Bundesregierung und der Bundesrat könnten Meinungsverschiedenheiten zu seiner Anwendung vielmehr durchaus vor das Bundesverfassungsgericht tragen. Es ging hier lediglich darum zu zeigen, daß sich ein imperatives Mandat des Ländervertreters kaum verfassungsgerichtlich, selbst im Wege des Organstreits, durchsetzen ließe. Ergänzend möchte ich noch die Möglichkeit ansprechen, daß ein imperatives Mandat des Ländervertreters irreal werden bzw. das Verhältnis zwischen Bundesrat und Ländervertreter sich umkehren könnte. Von Länderseite ${ }^{2 \mathrm{rT}}$ wird nämlich vorgeschlagen, daß der Ländervertreter im Sinne einer wirkungsvollen Wahrnehmung von Länderinteressen gegenüber Maßnahmen der Union möglichst frühzeitig auf europäischer Ebene tätig werden und mit der Bundesregierung Fühlung aufnehmen solle. Wenn er es für sinnvoll halte, solle er gar von sich aus eine Befassung des Bundesrats herbeiführen. Dann kann von einem imperativen Mandat seitens des Bundesrates kaum noch die Rede sein. Womöglich bleibt dem Bundesrat ganz im Gegenteil nur noch die Aufgabe, die Initiativen des Ländervertreters abzusegnen, bestenfalls oberflächlich zu kontrollieren.

Zusammenfassend machen die Interessenvertretung der Bundesländer und insbesondere das gewünschte imperative Mandat des Ländervertreters deutlich, wie schwierig es ist, Effizienz und Demokratie miteinander zu vereinbaren. Das gilt heute im Verhältnis der Bundesländer zur Europäischen Union wie bislang schon zum Bund. Angesichts dahinschmelzender eigener Kompetenzen bleibt den Ländern vielfach nur die Mitwirkung an der übergeordneten Normsetzung. Diese Mitwirkung aber legitimiert den Entzug der eigenen Zuständigkeiten.

\section{Die Zentralisierung von Länderkompetenzen - legitimiert durch Bun- desrat und Ländervertreter}

Die Verlagerung von Zuständigkeiten der Länder auf die Bundes- und ggf. die Unionsebene wird u. a. dadurch normalisiert und legitimiert - so läßt sich vermuten -, daß der Bundesrat sich laut Art. 23 V 2 und VI GG zu Angelegenheiten der Landesgesetzgebung äußert, was normalerweise überhaupt nicht zu seinen Aufgaben gehört (2). Im Rahmen einer übergreifenden Betrachtung stellt der Ländervertreter einen neuen Fall von Mitwirkungsföderalismus dar, von dem man sich eine Kompensation des zusammengeschrumpften Kompetenzföderalismus erhofft (3). Bevor ich diese beiden Gesichtspunkte erörtern werde, ist auf den engen Anwendungsbereich des Art. 23 VI GG einzugehen, der insofern nicht hält, was er zu versprechen scheint ( $\mathrm{I}$ ).

\section{Die minimale Zuständigkeit des Ländervertreters}

Ein Ländervertreter ist nur unter eng umgrenzten Voraussetzungen zu bestellen. Art. 23 VI I GG verlangt, daß rim Schwerpunkt ausschließliche Gesetzgebungsbefugnisse der Länder betroffen sind،. Diese Kompetenzen sind nicht sehr zahlreich: Es handelt

$20 \mathrm{Vgl}$. zu diesen Punkten Maunz/Schmidt-Bleibtreu/Klein/Ulsamer, Bundesverfassungsgertchtsgesetz. Kommentar, 1964 ff. (hier 1987), Rz. 16, 17, 23 zu $\$ 63$.

21 In unveröffentlichten Positionspapieren. 
sich um Fragen von Bildung und Kultur sowie um das Recht der polizeilichen Gefahrenabwehr. In diesen Bereichen wird die Europäische Union jedoch kaum tätig. Selbst wenn es entsprechende Vorhaben gibt, ist die Beteiligung eines Ländervertreters nicht zwingend, denn Art. 23 VI I GG ist lediglich eine Soll-Vorschrift. Zwei Ausnahmen nennt bereits das Durchführungsgesetz: Der Ländervertreter darf die Verhandlungsführung insoweit nicht ausüben, als die Bundesrepublik Deutschland den Vorsitz im Rat innehat. Er soll auch dann nicht tätig werden, wenn es um Tagesordnungspunkte geht, die der Rat ohne Aussprache genehmigt (\$6 III und IV). Die Bundesregierung hält es darüberhinaus für möglich, von der Bestellung eines Ländervertreters abzusehen aus Gründen politischer oder administrativer Opportunität oder wegen der Verpflichtung Deutschlands zu europarechtskonformem Verhalten. ${ }^{22}$ Sie eröffnet sich damit zahlreiche, jetzt noch gar nicht absehbare Schlupflöcher. Diese verschiedenen Ausnahmen bewirken auf deutscher Seite eine diskontinuierliche, zwischen Bundesregierung und Ländervertreter aufgesplitterte Verhandlungsführung und schwächen so die ohnehin geringe Autorität der tatsächlich tätigen Ländervertreter noch zusätzlich. Schließlich sei daran erinnert, daß dem Ländervertreter nur dann das letzte Wort gegen die Bundesregierung zusteht, wenn eine 2/3Mehrheit des Bundesrats es so will und keine finanziellen Belastungen des Bundes zu befürchten sind.

Alles in allem dürften die realen Einflußmöglichkeiten der Länder in Angelegenheiten der Europäischen Union zwar gewachsen, aber immer noch bescheiden sein. Eine oberflächliche Lektüre sowie die juristische und politische Diskussion des neuen Art. 23 GG vermitteln jedoch den gegenteiligen Eindruck. Wir müssen daher davon ausgehen, daß die Vorschriften über den Ländervertreter vorerst mehr legitimatorische als praktische Wirkungen haben.

\section{Die Beteiligung des Bundesrates in Bereichen der Landesgesetzgebung}

Die Benennung und Instruktion des Ländervertreters durch den Bundesrat ist verfassungsrechtlich nicht nur deshalb problematisch, weil dabei einzelne Landesregierungen überstimmt werden können. Auch bei einstimmiger Beschlußfassung erwüchsen Bedenken aus dem Umstand, daß das Bundesorgan Bundesrat auf dem Gebiet der Landesgesetzgebung innerstaatlich gar nichts zu suchen hat. Nach dem Grundgesetz wird der Bundesrat im Hinblick auf die Gesetzgebung und Verwaltung des Bundes tätig; darüberhinaus dient er den Ländern allerdings auch bei der Mitwirkung in Angelegenheiten der Europäischen Union (Art. 50 GG). Das Problem liegt letztlich darin, in welchem Verhältnis zwei der Bedingungen für die Bestellung eines Ländervertreters zueinander stehen, nämlich die Voraussetzung einer ausschließlichen Gesetzgebungskompetenz der Länder und die Voraussetzung, es müsse um Angelegenheiten der Europäischen Union gehen (wobei letzteres aus Art. 23 II I GG folgt). Es ließe sich argumentieren, daß diese beiden Bedingungen einander ausschließen. Werde die Union nämlich tätig, seien die Länder insoweit gar nicht mehr zuständig. Auch bevor die Union bzw. früher die drei Gemeinschaften auf einem bestimmten Gebiet Regelungen getroffen habe, insbesondere nach Art. 235 EG-Vertrag, hätten die Länder ihre diesbezüglichen Kompetenzen bereits verloren, und zwar seit Inkrafttreten der Gemeinschaftsverträge. Sie seien insoweit allenfalls subsidiär mangels europäischer Regelung tätig geworden. ${ }^{23}$

22 Gesetzesbegrindung (Fn. 3), S.9 f.

${ }_{3}$ Vgl. z. B. Tobias Busch, Bundeslander und Grundgesetz im Spannungsfeld der europauschen Integration, I990, S. $205-21$ I. 
Diese Argumentation ist mit dem Wortlaut des Art. 23 VI 1 GG nicht vereinbar. Denn da sich seine beiden Voraussetzungen ja ausschließen sollen, wäre niemals ein Ländervertreter zu bestellen. Ein solch unsinniges Ergebnis kann nicht gewollt sein. Deshalb wäre die Bedingung der ausschließlichen Landesgesetzgebungsbefugnis so auszulegen, daß es sich um Materien handeln muß, in denen die Länder innerstaatlich allein die Gesetzgebungskompetenz bätten. Diese Auslegung kann sich auf Art. 23 IV GG stützen, wonach der Bundesrat an der europapolitischen Willensbildung des Bundes u. a. dann zu beteiligen ist, soweit die Länder innerstaatlich zuständig wären<.

Es ist allerdings auch gar nicht ersichtlich, welches rechtlich beachtenswerte Interesse noch an einer Mitwirkung der Länder bestünde, wenn diese die fraglichen Kompetenzen ohnehin nur noch subsidiär und provisorisch ausübten. Außerdem stößt die oben genannte Argumentation noch auf Zweifel im Rahmen einer historischen Auslegung. Sie läßt nämlich außer Acht, daß den Gründerstaaten der drei Gemeinschaften keinesfalls eine so weitreichende Absicht unterstellt werden kann. Insbesondere ist nicht anzunehmen, daß die Bundesrepublik Deutschland ihre Bundesstaatlichkeit von vornherein in diesem Ausmaß eingeschränkt bzw. zur Disposition gestellt habe. Es ist deshalb davon auszugehen, daß die beiden oben genannten Voraussetzungen sich nicht gegenseitig ausschließen. Die Zuständigkeit der Europäischen Union begründet sich nach Europarecht, die Zuständigkeit der Länder beruht auf dem Grundgesetz. Daß sich die abstrakt abgesteckten Kompetenzbereiche überschneiden (wie ja auch bei der konkurrierenden Gesetzgebung zwischen Bund und Ländern), dürfte unschädlich sein, solange für den Fall der Kollision zweier konkreter Kompetenzwahrnehmungen eine Prioritätsregel existiert, also z. B. der Vorrang und die unmittelbare Geltung des Gemeinschaftsrechts oder das Subsidiaritätsprinzip. Demnach kann es auch in Angelegenheiten der Europäischen Union ausschließliche Gesetzgebungsbefugnisse der Länder geben.

Die Beteiligung des Bundesrates gem. Art. 23 V 2 und VI GG ist - im Unterschied zu den entsprechenden einfachgesetzlichen Regelungen des Art. 2 EEAG - deshalb zwar noch nicht verfassungswidrig. Denn sie tastet wohl nicht den von Art. 79 III GG geschützten Wesensgehalt des Föderalismusprinzips an. Aber die Bundesratsbeteiligung hat jedenfalls zur Folge, den konkreten Verlust von Gesetzgebungsbefugnissen der Länder an die Europäische Union (oder auch später in anderem Zusammenhang an den Bund) nach dem bundesdeutschen Recht zu legitimieren.

\section{Die erhoffte Kompensation des geschwächten Kompetenz-durch den Mitwirkungsföderalismus}

Seit Gründung der Bundesrepublik Deutschland haben die Länder im Rahmen der Vereinheitlichung der Lebensverhältnisse Zuständigkeiten an den Bund verloren. Zugleich hat der Bundesrat immer mehr an Bedeutung gewonnen: Der Anteil zustimmungspflichtiger Bundesgesetze liegt nicht, wie 1949 erwartet, bei $10 \%$, sondern bei nunmehr fast $60 \%{ }^{24}$ Diese beiden Entwicklungen dokumentieren die Hoffnung, der Kompetenzverlust der Länder könne durch ihre über den Bundesrat vermittelte Mitwirkung an der Bundesgesetzgebung ausgeglichen werden. Schlagwortartig gesagt: Der gewachsene Mitwirkungsföderalismus könne den zusammengeschmolzenen Kompetenzföderalismus kompensieren. Doch die Kompensation ist unvollständig, denn über den Bundesrat können sich nur die Landesregierungen, nicht aber die Landesparlamente einbringen.

${ }_{24}$ Christian Engel, Bericht zu Deutschland, in: J. Charpentier / J.-D. Mouton (Hg.), Les régions de l'espace communautaire, Presses universitaires de Nancy I 992, S.65 ff. (68 m. w. N.). 
Der Mitwirkungs- und Exekutivföderalismus hat das Bundesorgan Bundesrat in einen Ort der Interessenkoordination und -vertretung der Länder verwandelt. Darüberhinaus wurde aus einem Faktor der vertikalen Gewaltenteilung ein zusätzliches Instrument der parteipolitischen - also horizontalen - Gewaltenteilung. So haben die Landesregierungen mit Hilfe des Bundesrates die Zentralisierung des politischen Lebens nicht nur legitimierend begleitet, sondern auch selbst betrieben. Ihre Macht hat sich dabei lediglich verlagert, nicht vermindert. Wohl aber haben die föderale Vielfalt der Bundesrepublik und die parlamentarische Demokratie in den Ländern gelitten. Die vom Föderalismus auch bezweckte Machtmäßigung ist ebenfalls beeinträchtigt. Insofern entfaltet die antiparlamentarische Absicht, in der Bismarck die Ländervertretung geschaffen hatte, bis heute ihre Wirkung. ${ }^{25}$ Doch Alternativen sind nicht ersichtlich. Solange die wirtschaftlichen und politischen Verhältnisse sich vereinheitlichen und zentralisieren, sichert wahrscheinlich allein der exekutive Mitwirkungsföderalismus eine gewisse Eigenständigkeit der Länder.

Die Einrichtung eines Ländervertreters setzt die hier zusammengefaßte Entwicklung auf der Ebene der Europäischen Union fort. Den Verlust eigener Kompetenzen wollen die Länder durch Mitwirkung am Unionshandeln kompensieren. Wiederum steht ein bloßer exekutiver Mitwirkungsföderalismus auf dem Programm. Wiederum gibt es keine Alternativen. Insbesondere haben sich die Äußerungsmöglichkeiten der Länder außerhalb des Bundesrates als völlig untauglich erwiesen.

Die Zuständigkeiten des Ländervertreters sind bislang minimal. Insofern könnte man von einer Beruhigungspille sprechen. Doch ist hier eine Tür aufgestoßen worden, die längerfristig ganz neue Wege weist. Das Institut des Ländervertreters durchbricht das Monopol der Bundesregierung in der Außen- und Europapolitik. Die Länder können auf eine gewisse Stärkung in einem Europa der Regionen hoffen. Bleibt die Frage: Dokumentiert das oder dementiert das die Ansicht von Hans Kelsen, ein Föderalismus auf drei Ebenen - hier also Länder, Bund und Europa - sei auf Dauer nicht durchzuhalten ${ }^{26}$ ?

Deutscher Juristinnenbund e.V. (Hrsg.)

\section{Juristinnen in Deutschland}

Die Zeit von 1900 bis 1998

3., völlig neubearbeitete Auflage

Geschichte der Juristinnen in Deutschland, ihr Einfluß - insbesondere durch die Arbeit des Deutschen Juristinnenbundes - auf Rechtspolitik und Gesetzgebung; ergänzt durch ausgewählte Kurzbiographien.

1998, 227 S., brosch., 34,- DM, 248,- öS, 3/,50 sFr, ISBN 3-7890-56। I-1

(Schriftenreihe Deutscher Juristinnenbund e.V., Bd. 1)

2s Hermann Eicher, Der Machtverlust der Landesparlamente. Histortscher Ruckblick, Bestandsaufnabme, Reformansatze, 1988, S. 108.

26 Vgl. Hans Kelsen, Die staatsrechtluche Durchfubrung des Anschlusses Osterreuchs an das deutsche Rezch, Zeitschrift fur offentliches Recht 1927, S. 329 ff. (331). 